\title{
Performing ICSI within 4 hours after denudation optimizes clinical outcomes in ICSI cycles
}

Yini Zhang ${ }^{1}$, Yongzhuang $\mathrm{Ma}^{2}$, Zishui Fang ${ }^{1}$, Shiqiao $\mathrm{Hu}^{1}$, Zhou Li ${ }^{1}$, Lixia Zhu ${ }^{1 *}$ and Lei $\mathrm{Jin}^{1 *}$

\begin{abstract}
Background: The study aimed to investigate whether and how general and partial time intervals between processes, from human chorionic gonadotrophin (HCG) trigger to intracytoplasmic sperm injection (ICSI), affected the laboratory and reproductive outcomes in ICSI cycles.

Methods: This was a retrospective data analysis of 3602 women who underwent ICSI treatment cycles using partner or donor sperms, performed at Reproduction Medicine Center of Tongji Hospital of Tongji Medical College of Huazhong University of Science and Technology (Wuhan, China) between October 2016 and September 2018. The clinical pregnancy rate was the major outcome in the study. The fertilization and available embryo rates were secondary outcomes.

Results: Data from 3602 consecutive fresh ICSI cycles was analysed. Multivariate linear regression and logistic regression analysis of factors related to fertilization and clinical pregnancy rates showed that fertilization rate $(P=$ $0.001)$ and clinical pregnancy rate $(P=0.037)$ were significantly associated with denudation (DN)-ICSI interval. Long DN-ICSI interval was associated with higher rate of fertilization than short DN-ICSI interval but significantly decreased clinical pregnancy rate when the interval is over $4 \mathrm{~h}(P<0.05)$.

Conclusions: DN-ICSI time interval can act as an independent predictor for clinical outcomes in ICSI cycles. The optimal time for ICSI is within $4 \mathrm{~h}$ after oocyte denudation for excellent laboratory and reproductive outcomes in ICSI cycles.
\end{abstract}

Keywords: Clinical pregnancy rate, Denudation, Fertilization rate, ICSI, Interval

\section{Background}

Intracytoplasmic sperm injection (ICSI) was born to primarily solve the infertility problems due to male factors and failure in vitro fertilization (IVF) treatments. In order to get more successful ICSI outcomes, the operation and its optimal timings including oocyte pick up $(\mathrm{OPU})$, denudation $(\mathrm{DN})$ and ICSI in ICSI procedures have been investigated $[1,2]$.

\footnotetext{
* Correspondence: zhulixia027@163.com; leijintongjih@qq.com

${ }^{1}$ Reproductive Medicine Center, Tongji Hospital, Tongji Medicine College, Huazhong University of Science and Technology, 1095 JieFang Avenue, Wuhan 430030, People's Republic of China

Full list of author information is available at the end of the article
}

The viewpoint finds general acceptance that $\mathrm{DN}$ and ICSI should be performed for a while after OPU in order to get sufficient time for achieving the maturation procedure [1]. Because oocytes need some time to get complete maturity so that oocytes can be made full use of the somatic cell compartment. While, the reported optimal timings for DN and ICSI could be various between reproductive medicine centers and among patients [2].

Satoshi Mizuno et al. [1] showed the presence of intact cumulus cells during the preincubation period for ICSI should be considered as a critical factor in fertilization and embryonic development, therefore, oocytes needed

C C The Author(s). 2020 Open Access This article is licensed under a Creative Commons Attribution 4.0 International License, which permits use, sharing, adaptation, distribution and reproduction in any medium or format, as long as you give appropriate credit to the original author(s) and the source, provide a link to the Creative Commons licence, and indicate if changes were made. The images or other third party material in this article are included in the article's Creative Commons licence, unless indicated otherwise in a credit line to the material. If material is not included in the article's Creative Commons licence and your intended use is not permitted by statutory regulation or exceeds the permitted use, you will need to obtain permission directly from the copyright holder. To view a copy of this licence, visit http://creativecommons.org/licenses/by/4.0/ The Creative Commons Public Domain Dedication waiver (http://creativecommons.org/publicdomain/zero/1.0/) applies to the data made available in this article, unless otherwise stated in a credit line to the data. 
to incubate some time before denudation. But recently a series of studies about the murine cumulus cell-oocyte complex (COC) indicated that prolonging the oocyte culture with intact cumulus cells could induce apoptosis. That was why DN might be carried out as quickly as possible after oocyte pick up [3-5].

The traditional view is that the denudation operation may damage the cell membrane of the oocyte, which may be caused by the chemical damage of the hyaluronidase and the mechanical damage of the pipette. Because oocytes should recover for a period of time after denudation [2], it is reasonable to conduct ICSI for a while after denudation. Furthermore, incubation of oocytes before ICSI may induce cytoplasmic maturation, allowing oocytes to gain full activation and normal development potential, which may increase fertilization rate and pregnancy rate.

However, Catherme patrat et al. [6] reported the time between denudation and ICSI was negatively correlated with fertilization rate. With the prolongation of time interval between denudation and ICSI, the fertilization rate gradually decreased. It was recommended that sperm injection should be performed immediately after denudation.

Though so many researches have tried to confirm the optimal time for OPU, DN and ICSI in ICSI procedures [7-12], no consistent agreement has been achieved.

In order to obtain an optimal clinical outcome in ICSI cycles, we investigated the large cohort of successive ICSI instances to explore whether and how general and partial time intervals between processes, from human chorionic gonadotrophin (HCG) trigger to sperm microinjection recorded by the operator specifically, affected the laboratory and clinical outcomes in assisted reproductive technology (ART) treatments.

\section{Methods}

\section{Study population and ethical approval}

In the present retrospective study, data were reviewed from women who underwent ICSI treatment cycles using partner or donor sperms, performed at Reproduction Medicine Center in the Tongji Hospital between October 2016 and September 2018. Each of the patients signed written informed consent. This study conformed to the Declaration of Helsinki for Medical Research involving Human Subjects. Meanwhile, Institutional Review Board approval was acquired from the Ethical Committee. All patients had signed authorization to receive their clinical data for analysis later at the beginning of ICSI treatment cycles. Besides,in order to avoid bias in the analysis, we set the exclusion criteria as follows: (a) the patients who had few oocytes retrieved $(\leq 4)$; (b) the patients who had low fertilization rate $(\leq 25 \%)$; (c) women or partner with chromosomal abnormality; (d) the patients who used frozen-thawed oocytes or donor oocytes for fertilization; (e) the patients whose purpose were preimplantation genetic testing (PGT); (f) males with obvious sperm morphology abnormality; (g) sperms from either testicular sperm aspiration or microsurgical epididymal sperm aspiration.

\section{Protocol for controlled ovarian hyperstimulation $(\mathrm{COH})$}

All patients in our research underwent controlled ovarian hyperstimulation $(\mathrm{COH})$. Ovarian stimulation was carried out using follicle-stimulating hormone (FSH) (Gonal-F, EMD Serono, Rockland, MA, USA) combined with gonadotropin-releasing hormone $(\mathrm{GnRH})$ agonist (Decapeptyl; Ferring, Kiel, Germany) and antagonist (Cetrotide; Merck Serono, Darmstadt, Germany). Even, the methods of ovarian stimulation protocols in our reproduction medicine center contains $\mathrm{GnRH}$ agonist long protocol, GnRH agonist short protocol, GnRH antagonist protocol and luteal phase ovarian stimulation, as described elsewhere [13]. The ovarian stimulation protocols and the daily dose of FSH injection were performed according to female ages, ovarian reserve and various reaction to ovarian stimulation in previous cycles. Follicular developments were monitored with ultrasound scanning. Then $250 \mathrm{mg}$ of recombinant Human Chorionic Gonadotropin (hCG) (Ovidrel; Merck Serono, Darmstadt, Germany) was used to for ovulation triggering when three leading follicles reached a mean diameter of $17 \mathrm{~mm}$ or two leading follicles reached a mean diameter of $18 \mathrm{~mm}$. Normally, performing oocytes retrieval transvaginally was at least $36 \mathrm{~h}$ after HCG trigger. Additionally, embryos were transferred on Day3 and all the transferred embryos were at least six cells with even blastomeres and $<20 \%$ fragmentation.

\section{Timing control}

Exact times including HCG, OPU, DN and ICSI were recorded by the operator to establish a databank of exact times. The detailed times collected were analysed to evaluate the relationship of general and partial time intervals between processes, from HCG to sperm microinjection, and ICSI outcomes including the fertilization rate, available embryo rate and clinical pregnancy rate. In particular, we identified the time interval between HCG trigger and oocyte pick up as T1, oocyte pick up and denudation interval as T2, denudation and ICSI interval as T3, HCG trigger and denudation as T4, oocyte pick up and ICSI interval as T5 and HCG trigger and ICSI interval as T6 (Fig. 1).

\section{Outcome measure}

We regarded clinical pregnancy rate as the major outcome in our research. While, the fertilization and available embryo rates were secondary outcomes. Clinical 


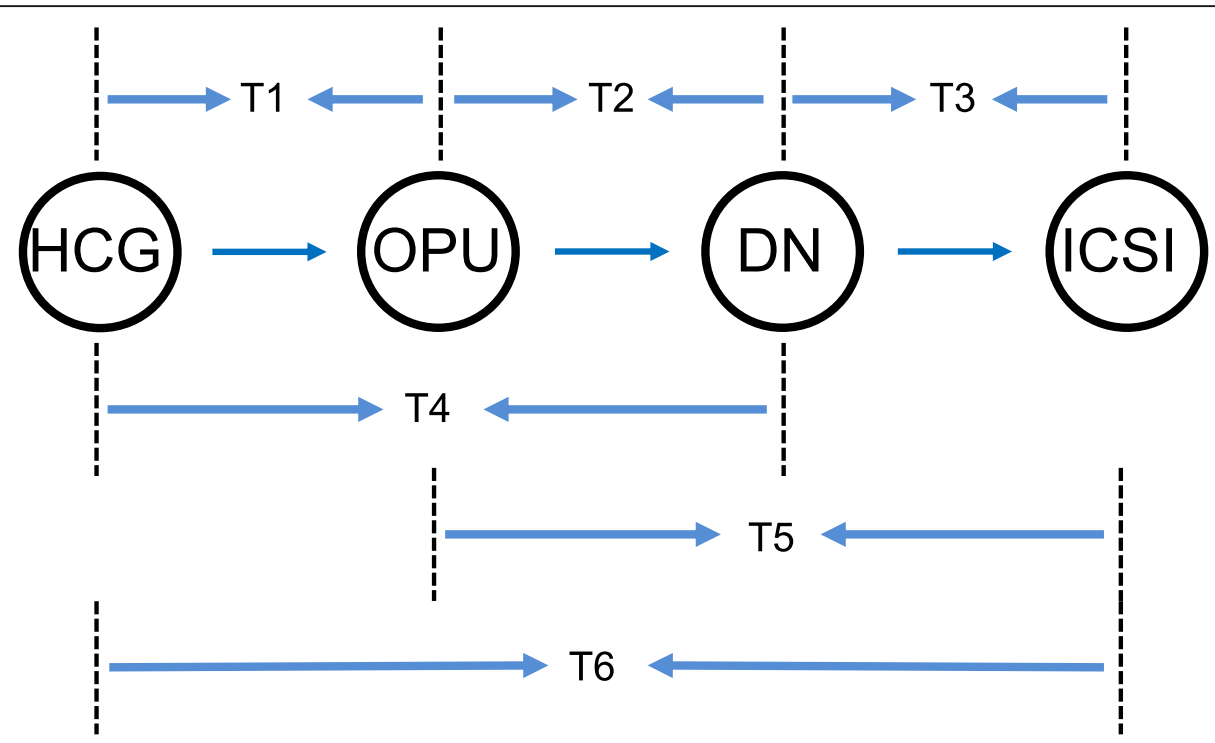

Fig. 1 General and partial time intervals between processes, from HCG trigger to ICSI

pregnancy was defined as the presence of a gestational sac with fetal heartbeat 4 weeks after transfer.

\section{Statistics}

SPSS 25.0 (IBM, Armonk, NY, USA) was used to analyze the study data and GraphPad Prism version 6 (GraphPad Software, La Jolla, CA, USA) was used for graph preparation. Moreover, correlation analysis, multivariate linear regression analysis and logistic regression analysis were used to study the effect of times on laboratory and reproduction rates in ICSI cycles. The interval between DN and ICSI varied between $0 \mathrm{~min}$ and $340 \mathrm{~min}$ (mean $166.51 \pm 60.29 \mathrm{~min}$ ). To rule out bias in the results by assuming that any relationship between DN-ICSI interval and the clinical pregnancy rate may be linear, patients were divided into five distinct groups according to the DN-ICSI intervals: $0-1.00$, $1.00-2.00,2.00-3.00,3.00-4.00,4.00-5.00$ and $>5.00 \mathrm{~h}$ for further analysis and confirmation. Equal intervals were set according to these cut-off points for exploring the threshold values. Additionally, associations between demographic and clinical characteristics of the patients were assessed with the use of Student $t$ test or Mann-Whitney $U$ test for continuous variables and chi-square for categoric variables, as appropriate. Results are expressed as mean \pm SD. $P$ value less than 0.05 was statistical significance for all data analysed.

\section{Results}

The study included a total of 3602 fresh ICSI cycles between October 2016 and December 2018. Table 1 demonstrates the demographic characteristics, cycle characteristics and reproductive outcomes of included cases. The mean age of women in the study was $30.76 \pm 4.73$ years (range $20-49$ years). The mean BMI of women in the study was
$21.91 \pm 4.68 \mathrm{~kg} / \mathrm{m} 2$. Percentage of primary infertility is $72.3 \%$. The mean duration of infertility was $3.79 \pm 2.84$ years. The mean basal FSH was $7.50 \pm 2.21 \mathrm{IU} / \mathrm{L}$. The mean number of oocytes retrieved was $13.36 \pm 6.23$. The fertilization and clinical pregnancy rates were 75.59 and $55.31 \%$, respectively.

Correlation analysis of T1-T6 associated with reproduction rates (Table 2) showed the $\mathrm{T} 3, \mathrm{~T} 5$ and $\mathrm{T} 6$ were significantly correlated with the fertilization rate and T3 was significantly correlated with the clinical pregnancy rate. By contrast, there was no significant correlation of $\mathrm{T} 1, \mathrm{~T} 2$ and $\mathrm{T} 4$ with: the fertilization or clinical pregnancy rates.

Table 1 Demographic characteristics, cycle characteristics, and reproductive outcomes of included cycles

\begin{tabular}{ll}
\hline Parameter & Value \\
\hline No. of cycles & 3602 \\
Age $(y)$ & $30.76 \pm 4.73$ \\
BMI $\left(\mathrm{kg} / \mathrm{m}^{2}\right)$ & $21.91 \pm 4.68$ \\
Percentage of primary infertility (\%) & 72.3 \\
Duration of infertility (y) & $3.79 \pm 2.84$ \\
Basal FSH (IU/L) & $7.50 \pm 2.21$ \\
No. of oocytes retrieved & $13.36 \pm 6.23$ \\
Fertilization rate (\%) & $75.59 \pm 17.85$ \\
Clinical pregnancy rate (\%) & 55.31 \\
HCG-OPU interval (min) & $2218.34 \pm 27.00$ \\
OPU-DN interval (min) & $130.60 \pm 46.53$ \\
DN-ICSI interval (min) & $166.51 \pm 60.29$ \\
\hline
\end{tabular}

Note: Data are presented as mean \pm SD or $n(\%)$ $B M I$ body mass index, OPU oocyte pick-up, DN denudation, ICSI intracytoplasmic sperm injection 
Table 2 Correlation analysis of factors related to the fertilization rate and clinical pregnancy rate in ICSI cycles

\begin{tabular}{llllll}
\hline \multirow{2}{*}{$\begin{array}{l}\text { Time } \\
\text { interval }\end{array}$} & \multicolumn{2}{l}{ Fertilization rate } & & \multicolumn{2}{l}{ Clinical pregnancy rate } \\
\cline { 2 - 3 } & Pearson C.C. & $P$ value & & Pearson C.C. & $P$ value \\
\hline T1 & 0.013 & 0.439 & & 0.023 & 0.279 \\
T2 & -0.018 & 0.285 & & 0.009 & 0.650 \\
T3 & 0.105 & $<0.001^{\mathrm{a}}$ & -0.042 & $0.042^{\mathrm{b}}$ \\
T4 & -0.013 & 0.430 & & 0.028 & 0.174 \\
T5 & 0.074 & $<0.001^{\mathrm{a}}$ & -0.029 & 0.169 \\
T6 & 0.088 & $<0.001^{\mathrm{a}}$ & -0.023 & 0.267 \\
\hline
\end{tabular}

Pearson C.C. Pearson correlation coefficients

${ }^{a}$ Correlation is significant $<0.001$ level

${ }^{\mathrm{b}}$ Correlation is significant $<0.05$ level

The multivariate linear regression and logistic regression analysis of factors related to the fertilization and clinical pregnancy rates is shown in Table 3 . The fertilization rate $(P=0.001)$ and clinical pregnancy rate $(P=0.037)$ were significantly related to DN-ICSI interval in ICSI cycles.

Figure 2 shows the overall association between DNICSI interval and the fertilization, available embryo and clinical pregnancy rates. In order to get detailed correlation, DN-ICSI time interval was divided into different parts: $0-1.00,1.00-2.00,2.00-3.00,3.00-4.00,4.00-5.00$ and $>5.00 \mathrm{~h}$. Obviously, there was a raise in the fertilization rate with longer DN-ICSI interval within $5 \mathrm{~h}$ and decline with progressively prolong DN-ICSI interval after $5 \mathrm{~h}$. In addition, the available embryo rate was higher within $0-2 \mathrm{~h}$ post $\mathrm{DN}$ and decreased thereafter. With an increase of time between DN and ICSI, clinical pregnancy rate decreased correspondingly progressively in ICSI cycles.

Furthermore, when DN-ICSI interval was within 4-5 $h$, the clinical pregnancy rate was significantly lower $(P<0.05)$ than DN-ICSI interval $<1.0 \mathrm{~h}$, also in DN-ICSI interval $>5 \mathrm{~h}$. Figure 2 also outlines there was an obvious decline of clinical pregnancy rate when DN-ICSI interval was $>4 \mathrm{~h}$. Therefore, in order to find an appropriate cutoff point, DN-ICSI interval of $2 \mathrm{~h}, 3 \mathrm{~h}$ and $4 \mathrm{~h}$ were referred as cut-off points to confirm the consequences. Meanwhile, for avoiding the effect of the number of embryos transferred on the clinical pregnancy rate, we

Table 3 Multivariate linear analysis and logistic regression analysis of factors related to the fertilization rate and clinical pregnancy rate in ICSI cycles

\begin{tabular}{lll}
\hline Time & $P$ value & \\
\cline { 2 - 3 } interval & Fertilization rate & Clinical pregnancy rate \\
\hline T3 & 0.001 & 0.037 \\
T5 & 0.219 & 0.281 \\
T6 & 0.314 & 0.130 \\
\hline
\end{tabular}

specially calculated the implantation rate between groups as assigned and found that there was no difference in the implantation rate of later two groups except the group in which cut-off point was $2 \mathrm{~h}$ (Fig. 3). Figure 3 demonstrates that clinical pregnancy rate was significantly different $(P<$ 0.05) in DN-ICSI interval $<4 \mathrm{~h}$ and $>4 \mathrm{~h}$. Thus, clinical pregnancy rate was significantly higher $(P<0.05)$ in DNICSI interval $<4 \mathrm{~h}$ compared to DN-ICSI interval $>4 \mathrm{~h}$ with similar implantation rates.

In order to clear up influence about oocyte aging because of different HCG trigger times on the relationship between DN-ICSI interval and reproductive outcomes, subgroup analysis according to HCG-OPU interval is exhibited in Fig. 4. All in all, the results further showed a negative correlation between prolonged DN-ICSI interval and the available embryo rate (Fig. 4).

\section{Discussion}

Our present study discovered DN-ICSI time interval acted as an independent predictor for clinical outcomes in ICSI cycles. Indeed, most investigators have showed the relation between DN-ICSI interval and fertilization rate $[6,11,14,15]$. Nevertheless, there are limited studies available for the influence on the clinical outcomes associated with raised DN-ICSI interval. Based on our findings, it is recommended performing ICSI within $4 \mathrm{~h}$ after denudation can optimize the clinical pregnancy rate in ICSI cycles.

The present study showed the increase in the fertilization rate over time of DN-ICSI interval. And the result was consistent with a recent large study [2] involving 1468 ICSI cycles which discovered the significant positive impact of the time before ICSI on fertilization. Also, Kubiak et al. [16] reported in mouse nuclear and cytoplasmic of oocytes maturation were distinguishable procedures. The process of the latter was referred as the capability of the oocyte to undergo complete activation. If performing ICSI before oocyte cytoplasm got maturation, meiosis might not resume and form of pronuclei might get a failure. Additionally, Rienzi et al. [7] showed that the optimal time for better outcomes of the fertilization rate in ICSI cycles appeared to be between 3 and $12 \mathrm{~h}$ after OPU, when oocytes tended to get complete cytoplasmic maturation.

Our results indicated that the increase of the fertilization rate might be caused by cytoplasmic maturation of immature oocytes and the stronger tendency of oocyte activation took about by in vitro culture, which might also result in ageing in vitro. Oocytes were usually fertilized without any delay following ovulation, and the window for appropriate fertilization time varied from species to species [1]. Normally if the oocyte did not be fertilized within the optimal interval, unfertilized oocyte could stay in the oviduct or culture medium, where it underwent a time-dependent decline in quality. And the 

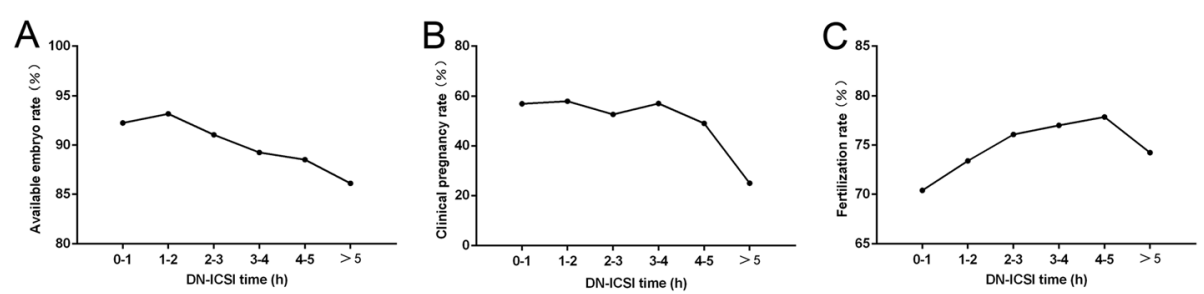

Fig. 2 The overall association between the DN-ICSI interval and fertilization rate, available embryo rate and clinical pregnancy rate

procedure was called "oocyte aging," which was referred as a main reason of compromised embryonic development following IVF, ICSI, and parthenogenetic activation $[17,18]$.

Additionally, it was found that the oocyte aging procedure was associated with changes in activity of Mphase promoting factor (MPF) [19], mitogen-activated protein kinase (MAPK) [20], concentration of calcium ions [21], and reactive oxygen species [22]. MPF was a protein kinase that played a regulatory role in oocyte meiosis and could promote progression into meiosis II [23]. Also, MPF maintained the high of activity state during the metaphase II. Besides MPF, metaphase IIarrested oocytes were exhibited by elevated levels activity of MARK which regulated the transition from meiosis I to meiosis II. In particular, both MPF and MARK were necessary in metaphase II stage arrest [24].

Carla et al. [25] observed changes of MPF and MAPK activities which showed spontaneous decrease after ovulation in-vitro culture. Kikuchi et al. [26, 27] found that along with oocytes aging, MPF converted to preMPF which had no activity of MPF because of the phosphorylation of cycline $\mathrm{B}$ and cycline-dependent kinase p34cdc2 complex. With prolonged incubation time in vitro, some oocytes were spontaneously activated which was the main manifestations of oocyte ageing due to the mode of MPF inactivation and decreasing MAPK activity [28]. In short, these changes made oocytes have 'activation competent'. Thus, aged oocytes with less MPF might be easily activated in ICSI treatments and form pronuclei. That is the reason for an increase of fertilization rate with increasing OPU-ICSI interval [2].

Opinions about the optimal time to perform ICSI following DN varied in different researches. In 1998, van de velde et al. [10] showed that a period of time between OPU and ICSI was not related to ICSI outcomes and MII oocytes may not require further cytoplasmic maturation. Also, Jacobs et al. [14] found that the denudation
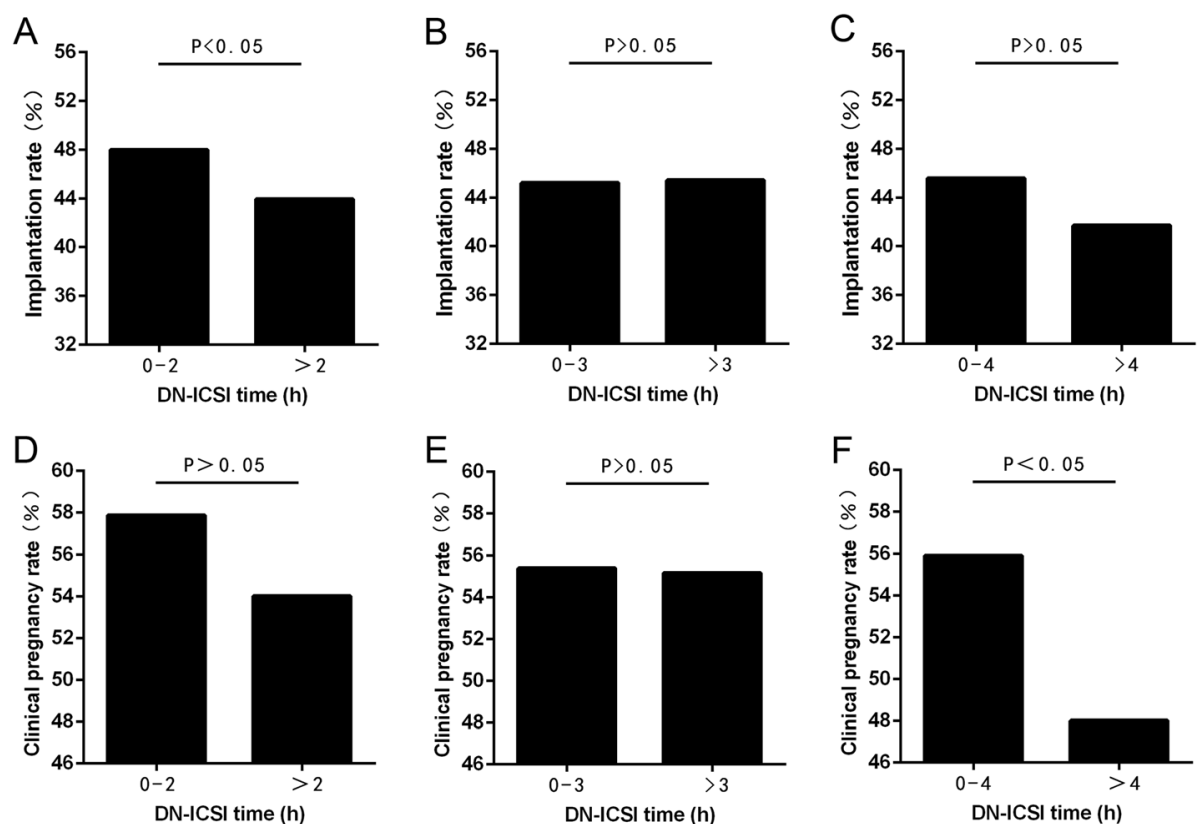

Fig. 3 Implantation rate differs according to DN-ICSI time cut-off points. a DN-ICSI time cut-off point,2 h; b DN-ICSI time cut-off point,3 h; c DNICSI time cut-off point, $4 \mathrm{~h}$. Clinical pregnancy rate differs according to DN-ICSI time cut-off points. d DN-ICSI time cut-off point,2 h; e DN-ICSI time cut-off point,3 h;(F)DN-ICSI time cut-off point,4 h 
A

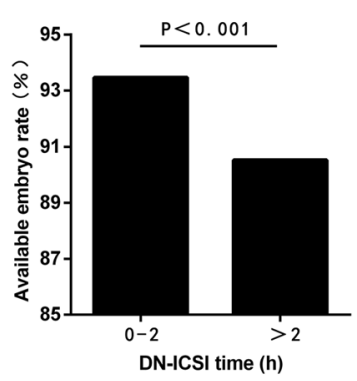

B

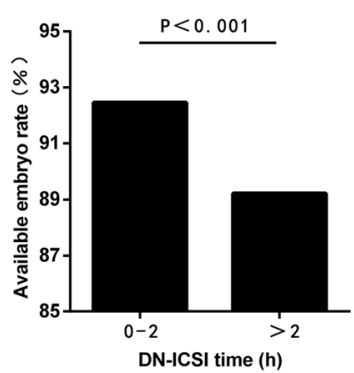

C

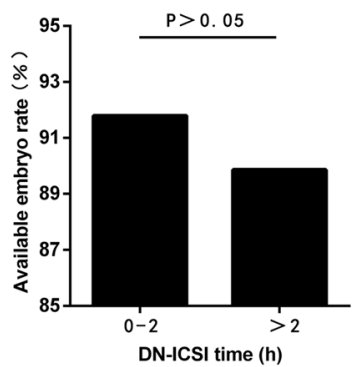

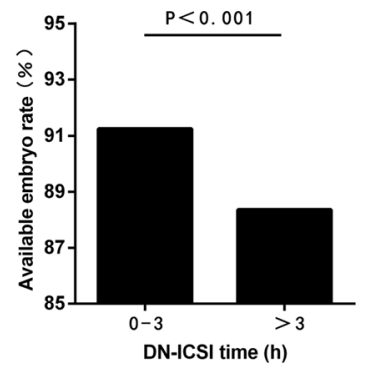
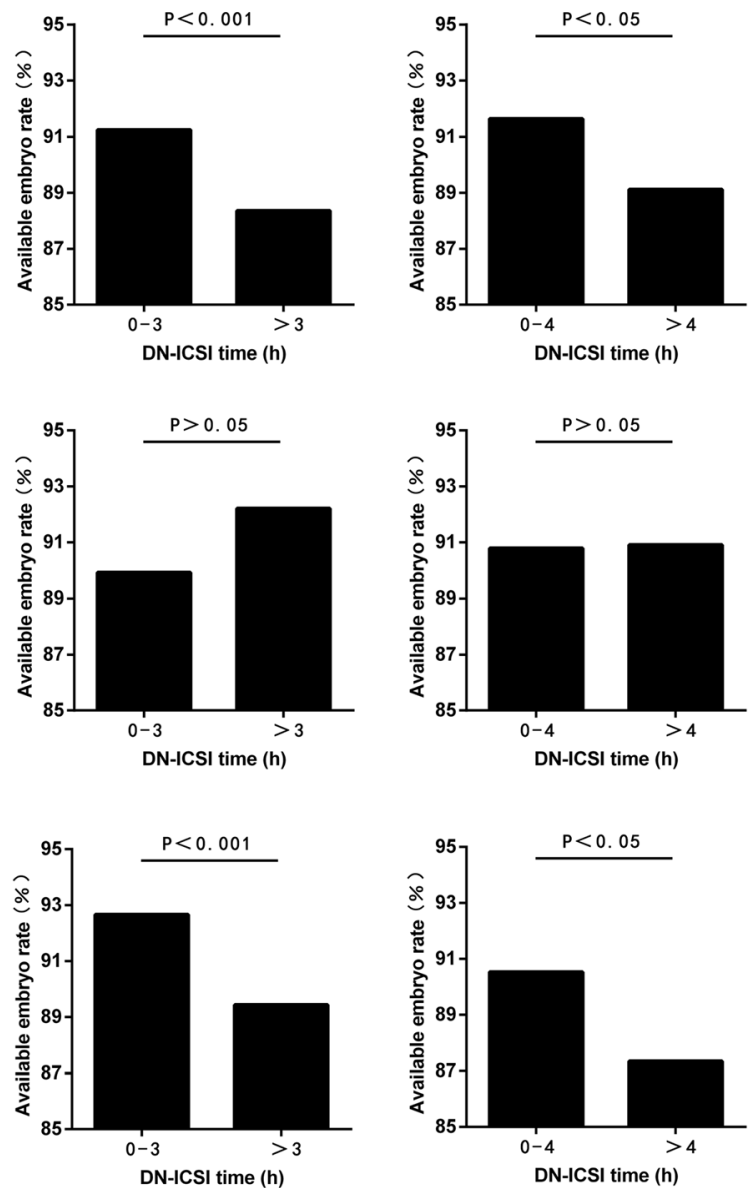

Fig. 4 Subgroup analysis between DN-ICSI interval and available embryo rate according to HCG-OPU interval. a When HCG-OPU interval was < 37 h; b When HCG-OPU interval was 37-38 h; c When HCG-OPU interval was > $38 \mathrm{~h}$

and ICSI interval was not related to the ICSI outcomes. On the contrary, Boldi et al. [15] retrospectively analyzed 203 ICSI cycles, which divided into $<1 \mathrm{~h}$ group and 1-3 $\mathrm{h}$ group according to time between DN and ICSI in 2010. The data indicated that the top-quality embryo, implantation and pregnancy rates of $<1 \mathrm{~h}$ group were higher than 1-3 h group, while fertilization rate was not statistically different between groups. It was believed that the procedure might achieve a better outcome if ICSI was performed immediately after denudation. Besides, Catherme patrat et al. [6] performed logistic regression analysis on 110 cycles and found that in terms of the fertilization rate, an optimal time to carry out DN was within $3 \mathrm{~h}$ following OPU and performing ICSI soon after DN was a good choice. However, other researches did not confirm the optimal time to perform ICSI following DN.

In the present study, we chose the clinical pregnancy rate as the main outcome chosen for analysis. Interestingly, we found an opposite tendency in fertilization and clinical pregnancy rates as DN-ICSI interval increased which indicated the negative correlation of DN-ICSI interval and the clinical pregnancy rate in ICSI cycles. As previously stated, if oocytes which should have be fertilized did not be fertilized within the appropriate period, the oocytes remained within the oviduct and culture medium [29, 30]. And the oocytes would suffer aging which impaired oocyte ability. In order to find an equilibrium timing point between full cytoplasmic maturation and oocyte aging, equally, the fertilization and clinical pregnancy rate, we set different cut-off points. The implantation rate corrected for imbalance in the number of embryos transferred across groups. More importantly, the data of clinical pregnancy rate in our research further clarified the conclusions that clinical pregnancy rate was significantly higher in DN-ICSI interval $<4 \mathrm{~h}$ compared to DN-ICSI interval $>4 \mathrm{~h}$ with similar implantation rates. Especially, the interval also seemed to be reasonable for fertilization rate.

The reason for the decline in the clinical pregnancy rate over time is what we want to know. Aging after ovulation seemed to influence oocyte function including 
oocyte growth and recruitment, alteration, expression and storage of mature factors. These limitations influenced the developmental capacity [31]. Undoubtedly mature oocytes owned separate compartments such as the subcortical RNP domain (SCRD) and spindle chromosome complex (SCC) for storing proteins and information [31, 32]. Furthermore, RNA-binding proteins (MSY2) which was abound in the SCRD and SCC significantly reduced because of oocyte aging might disrupt the recruitment, deadenylation and degradation of developmentally important RNAs. What is more, change in the SCC-MSY2 domain and the maternal effect protein BRG1 in chromatin potentially might have an effect on spindle integrity and normal epigenetic histone modifications. In particular, the absence of H3K9 trimethylation which was an important part in histone modifications and essential for bipolar spindle formation and chromosome alignment, might result in a risk of aneuploidy [33] and the decrease of clinical pregnancy rate.

Considering that HCG administration time was the key factor for oocyte final maturation [34] which further affected denudation and ICSI timing, we also performed a subgroup analysis according to HCG-OPU interval. And the result demonstrated that when ensuring a certain number of available embryos, $4 \mathrm{~h}$ was still the most optimal demarcation point taking into account both HCG-OPU interval and DN-ICSI interval to obtain better clinical outcomes.

As one limitation, implantation was not considered as a primary outcome index in our study because of the different number of embryos transferred in the enrolled patients. Since implantation potential was a more accurate indicator for oocyte vitality, in our future work, we will aim to explore a correlation between DN-ICSI internal and embryo implantation chance in a welldesigned single-embryo-transfer cohort study.

Compared with other available literatures, our study should be more convinced. As we know, distinct oocyte or sperm genetic disease greatly affect results, we set exclusion criteria to carry on an accurate analyze and avoid bias for the study. What's more, another strength is that our research includes the selection of a great number of infertile patients analysed, valid comparisons of outcomes in the cycles of different groups and detailed times from HCG to ICSI recorded by the exact operator independently. In addition, we also adopt measures of subgroup analysis to proclaim the relationship between DN-ICSI and available embryo rates in terms of an interval between HCG trigger and oocyte retrieval.

\section{Conclusions}

In conclusion, this study indicates that DN-ICSI interval significantly affects the clinical outcomes and the optimal timing for ICSI is within $4 \mathrm{~h}$ after oocyte denudation for excellent laboratory and reproductive results in ICSI cycles. DN-ICSI time interval can act as an independent predictor for clinical outcomes in ICSI cycles.

\section{Abbreviations \\ COC: Cumulus cell-oocyte complex; $\mathrm{COH}$ : Controlled ovarian \\ hyperstimulation; DN: Denudation; FSH: Follicle-stimulating hormone; HCG: Human chorionic gonadotropin; ICSI: Intracytoplasmic sperm injection; MPF: M-phase promoting factor; MAPK: Mitogen-activated protein kinase; OPU: Oocyte pick up}

\section{Acknowledgements}

We would like to extend science gratitude to our colleagues who work at our reproductive medicine center for their direct and indirect help to the project.

\section{Authors' contributions}

$J \mathrm{~L}$ and $\mathrm{ZLX}$ participated in designing this study and data acquisition. ZYN was involved in drafting the paper and analysing data. MYZ took part in proposing revisions and analysis of data. FZS provided several advices with statistical methods. Meanwhile, he participated in the revision of manuscript. HSQ supplied opinions of revision and critical discussions. LZ coordinated the study and revised the manuscript critically. The authors above have read this manuscript and allow to submit the final version for publication. All authors read and approved the final manuscript.

\section{Authors' information}

$L$, PHD, doctoral supervisor and professor of Obstetrics and Gynecology at the Huazhong University of Science and Technology and Director of the Assisted Reproductive Technologies Centre at Tongji Hospital, China. He conducts the research of male and female infertility, endometriosis and gynecological endocrine diseases.

\section{Funding}

The research acquired financial support from the National Science and Technology Major Project of China (2018YFC1002103).

Availability of data and materials

All data from the current study supporting the conclusions are presented in the article.

Ethics approval and consent to participate

This research was approved by the Ethical Committee of Tongji Hospital.

\section{Consent for publication}

Each of the patients signed written informed consent for publication.

\section{Competing interests}

The authors declare that they have no competing interests.

\section{Author details}

${ }^{1}$ Reproductive Medicine Center, Tongji Hospital, Tongji Medicine College, Huazhong University of Science and Technology, 1095 JieFang Avenue, Wuhan 430030, People's Republic of China. ${ }^{2}$ Department of Orthopedics, Tongji Hospital, Tongji Medical College, Huazhong University of Science and Technology, Wuhan, Hubei, People's Republic of China.

Received: 5 October 2019 Accepted: 2 April 2020

Published online: 14 April 2020

\section{References}

1. Mizuno S, Ishikawa Y, Matsumoto $H$, Sato M, Ida M, Fukuda A, Morimoto Y The timing of cumulus cell removal for intracytoplasmic sperm injection influences the capability of embryonic development. Reprod Med Biol. 2019;18:111-7.

2. Pujol A, Garcia D, Obradors A, Rodriguez A, Vassena R. Is there a relation between the time to ICSI and the reproductive outcomes? Hum Reprod. 2018;33:797-806.

3. Miao YL, Liu XY, Qiao TW, Miao DQ, Luo MJ, Tan JH. Cumulus cells accelerate aging of mouse oocytes. 3. Biol Reprod. 2005;73:1025-31. 
4. Qiao TW, Liu N, Miao DQ, Zhang X, Han D, Ge L, Tan JH. Cumulus cells accelerate aging of mouse oocytes by secreting a soluble factor(s). Mol Reprod Dev. 2008;75:21-8.

5. Zhu J, Zhang J, Li H, Wang TY, Zhang CX, Luo M, Tan JH. Cumulus cells accelerate oocyte aging by releasing soluble Fas ligand in mice. Sci Rep. 2015;5:8683.

6. Patrat C, Kaffel A, Delaroche L, Guibert J, Jouannet P, Epelboin S, De Ziegler $D$, Wolf JP, Fauque P. Optimal timing for oocyte denudation and intracytoplasmic sperm injection. Obstet Gynecol Int. 2012;2012:403531.

7. Rienzi L, Ubaldi F, Anniballo R, Cerulo G, Greco E. Preincubation of human oocytes may improve fertilization and embryo quality after intracytoplasmic sperm injection. Hum Reprod. 1998;13:1014-9.

8. Plachot M, Belaisch-Allart J, Mayenga JM, Chouraqui A, Tesquier L, Serkine AM. Outcome of conventional IVF and ICSI on sibling oocytes in mild male factor infertility. Hum Reprod. 2002;17:362-9.

9. Ho JY, Chen MJ, Yi YC, Guu HF, Ho ES. The effect of preincubation period of oocytes on nuclear maturity, fertilization rate, embryo quality, and pregnancy outcome in IVF and ICSI. J Assist Reprod Genet. 2003;20:358-64.

10. Yanagida K, Yazawa H, Katayose H, Suzuki K, Hoshi K, Sato A. Influence of oocyte preincubation time on fertilization after intracytoplasmic sperm injection. Hum Reprod. 1998;13:2223-6.

11. Van de Velde H, De Vos A, Joris H, Nagy ZP, Van Steirteghem AC. Effect of timing of oocyte denudation and micro-injection on survival, fertilization and embryo quality after intracytoplasmic sperm injection. Hum Reprod. 1998;13:3160-4

12. Hassan HA. Cumulus cell contribution to cytoplasmic maturation and oocyte developmental competence in vitro. J Assist Reprod Genet. 2001;18: 539-43.

13. Xu B, Chen Y, Geerts D, Yue J, Li Z, Zhu G, Jin L. Cumulative live birth rates in more than 3,000 patients with poor ovarian response: a 15-year survey of final in vitro fertilization outcome. Fertil Steril. 2018:109:1051-9.

14. Jacobs M, Stolwijk AM, Wetzels AM. The effect of insemination/injection time on the results of IVF and ICSI. Hum Reprod. 2001;16:1708-13.

15. Boldi CP, Colasante C, Perego L. Effect of early and late oocyte denudation on ICSI outcome. Hum Reprod. 2010;25(20100600):180.

16. Kubiak JZ. Mouse oocytes gradually develop the capacity for activation during the metaphase II arrest. Dev Biol. 1989;136:537-45.

17. Lacham-Kaplan O, Trounson A. Reduced developmental competence of immature, in-vitro matured and postovulatory aged mouse oocytes following IVF and ICSI. Reprod Biol Endocrinol. 2008;6:58.

18. Mizutani E, Jiang JY, Mizuno S, Tomioka I, Shinozawa T, Kobayashi J, Sasada $H$, Sato E. Determination of optimal conditions for parthenogenetic activation and subsequent development of rat oocytes in vitro. 8. J Reprod Dev. 2004;50:139-46.

19. Jiang GJ, Wang K, Miao DQ, Guo L, Hou Y, Schatten H, Sun QY. Protein profile changes during porcine oocyte aging and effects of caffeine on protein expression patterns. PLoS One. 2011;6:e28996.

20. Miao YL, Kikuchi K, Sun QY, Schatten H. Oocyte aging: cellular and molecular changes, developmental potential and reversal possibility. Hum Reprod Update. 2009;15:573-85.

21. Yoon SY, Fissore RA. Release of phospholipase C zetaand [Ca2+] i oscillation-inducing activity during mammalian fertilization. Reproduction (Cambridge, England). 2007;134:695-704.

22. Goud AP, Goud PT, Diamond MP, Gonik B, Abu-Soud HM. Reactive oxygen species and oocyte aging: role of superoxide, hydrogen peroxide, and hypochlorous acid. Free Radic Biol Med. 2008;44:1295-304.

23. Dekel N. Protein phosphorylation/dephosphorylation in the meiotic cell cycle of mammalian oocytes. Rev Reprod. 1996;1(2):82-8.

24. Fissore RA, Kurokawa M, Knott J, Zhang M, Smyth J. Mechanisms underlying oocyte activation and postovulatory ageing. Reproduction (Cambridge, England). 2002;124(6):745-54.

25. Tatone C, Carbone MC, Gallo R, Delle Monache S, Di Cola M, Alesse E, et al. Age-associated changes in mouse oocytes during postovulatory in vitro culture: possible role for meiotic kinases and survival factor BCL2. Biol Reprod. 2006;74(2):395-402.

26. Kikuchi K, Naito K, Noguchi J, Shimada A, Kaneko H, Yamashita M, et al. Inactivation of p34cdc2 kinase by the accumulation of its phosphorylated forms in porcine oocytes matured and aged in vitro. Zygote (Cambridge, England). 1999;7(2):173-9.

27. Kikuchi K, Naito K, Noguchi J, Shimada A, Kaneko H, Yamashita M, Aoki F, Tojo H, Toyoda Y. Maturation/M-phase promoting factor: a regulator of aging in porcine oocytes. Biol Reprod. 2000;63:715-22.
28. Petrova I, Chmelikova E, Svestkova D, Rajmon R. In vitro ageing of porcine oocytes. Czech J Anim Sci. 2004:49:93-8.

29. Mizutani E, Jiang JY, Mizuno S, Tomioka I, Shinozawa T, Kobayashi J, Sasada $\mathrm{H}$, Sato E. Determination of optimal conditions for parthenogenetic activation and subsequent development of rat oocytes in vitro. J Reprod Dev. 2004:50:139-46.

30. Bianchi S, Macchiarelli G, Micara G, Linari A, Boninsegna C, Aragona C, Rossi G, Cecconi S, Nottola SA. Ultrastructural markers of quality are impaired in human metaphase II aged oocytes: a comparison between reproductive and in vitro aging. J Assist Reprod Genet. 2015;32:1343-58.

31. Trapphoff $T$, Heiligentag M, Dankert D, Demond H, Deutsch D, Frohlich T, Arnold GJ, Grummer R, Horsthemke B, Eichenlaub-Ritter U. Postovulatory aging affects dynamics of mRNA, expression and localization of maternal effect proteins, spindle integrity and pericentromeric proteins in mouse oocytes. Hum Reprod. 2016:31:133-49.

32. Zhu K, Yan L, Zhang X, Lu X, Wang T, Yan J, Liu X, Qiao J, Li L. Identification of a human subcortical maternal complex. Mol Hum Reprod. 2015;21:320-9.

33. Bultman SJ, Gebuhr TC, Pan H, Svoboda P, Schultz RM, Magnuson T. Maternal BRG1 regulates zygotic genome activation in the mouse. Genes Dev. 2006;20:1744-54

34. Farrag A, Costantini A, Manna C, Grimaldi G. Recombinant HCG for triggering ovulation increases the rate of mature oocytes in women treated for ICSI. J Assist Reprod Genet. 2008;25:461-6.

\section{Publisher's Note}

Springer Nature remains neutral with regard to jurisdictional claims in published maps and institutional affiliations.
Ready to submit your research? Choose BMC and benefit from:

- fast, convenient online submission

- thorough peer review by experienced researchers in your field

- rapid publication on acceptance

- support for research data, including large and complex data types

- gold Open Access which fosters wider collaboration and increased citations

- maximum visibility for your research: over $100 \mathrm{M}$ website views per year

At $\mathrm{BMC}$, research is always in progress.

Learn more biomedcentral.com/submissions 